\title{
Relaciones del Origen del Tronco Celiaco, Arterias Mesentéricas y Renales con la Columna Vertebral en Individuos Chilenos
}

\author{
Relationships Between of the Origin of Celiac Trunk, Mesenteric \\ and Renal Arteries with the Vertebral Column in Chilean Individuals
}

*Puelma, F. \& ** Olave, E.

PUELMA, F. \& OLAVE, E. Relaciones del origen del tronco celiaco, arterias mesentéricas y renales con la columna vertebral en individuos chilenos. Int. J. Morphol., 28(4):1227-1234, 2010.

RESUMEN:En la actualidad muchos procedimientos quirúrgicos invasivos, diagnósticos y terapéuticos requieren de un conocimiento preciso de la anatomía arterial del territorio abdominal. Entre estas intervenciones destaca la extracción de órganos en donantes vivos (renal y hepático) y las intervenciones oncológicas, entre otras. Tradicionalmente, el estudio de los vasos se ha hecho por medio de arteriografías que requieren de procedimientos invasivos con inyección de medio de contraste y largas exposiciones a rayos X, obteniendo imágenes bidimensionales en las cuales las relaciones entre los tejidos y órganos es difícil de analizar. El objetivo del presente trabajo fue determinar las características anatómicas del tronco celiaco, arterias mesentérica superior, renales y mesentérica inferior y la relacion de origen con la columna vertebral. Se estudiaron las imágenes de 74 pacientes, 40 hombres y 34 mujeres, sometidos a una angiotomografía de la parte abdominal de la aorta, registrando las características y las relaciones de las arterias mencionadas. Las imágenes utilizadas se obtuvieron mediante tomografía computarizada helicoidal multidetectores. La división clásica del tronco celíaco en tres ramas se encontró en el 60,8\% de los casos, el origen se relacionó con T12 en 50\% de los hombres y en 52,9\% de las mujeres, con L1 en 50\% y 47,1\%, respectivamente. La a. mesentérica superior se originó a nivel de T12, L1 y L2 en un 8,1\%, 78,4\% y 13,5\%, respectivamente. Las aa. renales fueron dobles en el $18,9 \%$ de los casos. Con respecto a las descripciones clásicas encontramos diferencias que demuestran la variabilidad de la distribución vascular. El método se valida como una instancia útil de bajo riesgo para el paciente y reproducible por los investigadores y clínicos.

PALABRAS CLAVE: Anatomía; Parte abdominal de la aorta; Tomografía computarizada helicoidal.

\section{INTRODUCCIÓN}

La aorta abdominal durante su trayecto emite numerosas ramas que se pueden clasificar de acuerdo a las estructuras que irrigan, ramas parietales o ramas viscerales, considerando ramas parietales a las que irrigan la pared abdominal y viscerales a aquellas que irrigan vísceras intraabdominales o extrabdominales tales como los genitales masculinos (Testut \& Latarjet, 1969).

En la actualidad, con el advenimiento de terapias cada vez menos invasivas, con procedimientos diagnósticos, quirúrgicos y terapéuticos que requieren de mayor precisión y accesos mínimos, se requiere de un conocimiento anatómico exacto de la posición, relaciones y ramas de la aorta abdominal.

* Facultad de Medicina, Universidad Católica del Maule, Talca, Chile.

** Facultad de Medicina, Universidad de La Frontera, Temuco, Chile.

Programa de Magíster en Ciencias, Mención Morfología, Universidad de La Frontera, Chile.
En el 2004, Tooher et al. señalaron que en el trasplante renal - cuando se utiliza un donante vivo- se requiere conocer con absoluta certeza la localización y variaciones de las arterias renales, ya que el procedimiento de extracción se hará por vía laparoscópica como método quirúrgico de elección, por ser la mejor alternativa para el donante. Cuando se procede a la extracción de múltiples órganos en un donante cadáver, el cirujano debe hacer una amplia disección de todo el retroperitoneo exponiendo los grandes vasos y en especial disecando y reconociendo la aorta y sus ramas, ya que es requisito para proceder a perfundir los diferentes órganos intraabdominales para trasplante.

Durante las cirugías oncológicas se debe resecar los 
diferentes grupos de nodos linfáticos los cuales en su gran mayoría se ubican en el trayecto de importantes ramas de la aorta tales como el tronco celíaco (Wang et al., 2009).

Por su parte, Tovar et al. (1999) señalaron que como método diagnóstico se ha utilizado ampliamente la aortografía translumbar, lo que requiere de un conocimiento acabado de las relaciones y puntos importantes del trayecto aórtico a fin de evitar lesiones o iatrogenias.

Actualmente, el trauma es la primera causa de muerte en personas jóvenes, en especial hombres y dentro de estos traumas, reconocemos los traumas abdominales abiertos o penetrantes (por arma blanca) como una de las causa mas frecuentes que son motivos de consultas en los servicios de emergencia. En las heridas penetrantes abdominales es primordial conocer el trayecto de la aorta abdominal y sus ramas, ya que permite sospechar y tratar lesiones que, si no son tratadas, generalmente son letales o dejan severas secuelas. Existen otros múltiples ejemplos de procedimientos en que la anatomía de la aorta abdominal es clave para el éxito de los procedimientos (Rubin et al., 1993 a).

Disponemos de múltiples textos de anatomía clásica en la cual se hacen detalladas descripciones de la aorta abdominal, de sus ramas y de sus relaciones (Testut \& Latarjet; Williams et al., 1995; Moore \& Dalley, 2004). Estas descripciones resultan de sumo interés y son producto de cuidadosas y múltiples disecciones. Sin embargo, diferentes técnicas de disección y mediciones subjetivas hacen que algunas de estas descripciones carezcan de validez para la precisión que requieren procedimientos médicos actualmente utilizados.

Los métodos radiológicos clásicos, sin duda muy útiles, muchas veces daban información relevante pero con baja capacidad de discriminación y hacían poco precisas las descripciones y relaciones entre los órganos estudiados. Posteriormente, se desarrollaron técnicas radiológicas vasculares, inyectando un medio de contraste en la estructura a estudiar, permitiendo un mejor conocimiento anatómico, pero significando un gran trauma para el paciente y sobre todo la posibilidad de un severo daño por la nefrotoxicidad de estos medios (Cikrit et al. 1996; Mautone \& Brown, 2010).

A fines del siglo pasado y sobre todo al principio del siglo XXI, hemos asistido a la utilización del desarrollo cibernético y su aplicación a múltiples áreas en diversos territorios del ámbito médico (Rubin et al., 1993b). La aplicación en el área de imagenología ha permitido obtener imágenes anatómicas de todo el cuerpo humano, permitiendo describir y estudiar sus características desde un nivel macro hasta el ultraestructural.
Este desarrollo ha llevado a conocer la tomografía axial computarizada (TAC) helicoidal, en especial la angiotomografía axial computarizada, donde las imágenes resultantes del análisis y procesamiento computacional que efectúa el tomógrafo son muy claras y definidas (Beebe \& Kritpracha, 2003). Esta metodología de obtención de imágenes volumétricas con definiciones milimétricas permite hacer reconstrucciones en tres dimensiones y relacionarlas con los diferentes tejidos.

Basado en lo anterior, nos propusimos determinar en un grupo de individuos chilenos, las características anatómicas de ramas viscerales de la aorta, determinando la presencia, origen, variaciones y relaciones respecto a la columna vertebral. Las arterias consideradas fueron el tronco celíaco, a. mesentérica superior, aa. renales y a. mesentérica inferior.

\section{MATERIAL Y MÉTODO}

Se estudiaron angiotomografías obtenidas en forma consecutiva en 74 personas, de nacionalidad Chilena, de ambos sexos, 40 hombres (54\%) y 34 mujeres ( $46 \%$ ), con edades comprendidas entre 6 y 84 años, con un promedio de 29 años.

Las angiotomografías helicoidales se realizaron a personas que se encontraban con indicación de estudio vascular abdominal en el Servicio de Radiología del Hospital Regional de Talca, Chile.

Para los registros se utilizó un tomógrafo computacional multidetector de 16 detectores, General Electric, con un protocolo de adquisición 32 × 1,25 canales por milímetro, pitch 1.375 , velocidad de mesa 55 milímetros por segundo, en un tiempo de 5,5 segundos.

Se utilizó un protocolo de adquisición con una fase arterial temprana (20 segundos) y una fase arterial tardía (30 - 35 segundos), no se utilizó la fase tardía para estudio venoso (60 segundos). Se utilizó una duración de escaneo de 10 y 5 segundos para la fase precoz y tardía, respectivamente, con retraso de 22 y 40 segundos para cada fase respectivamente. La velocidad de inyección del medio de contraste fue de $4,5 \mathrm{ml} / \mathrm{segundo}$.

En cada una de ellas, se registraron las variables siguientes: nombre, sexo, edad y la relación del tronco celíaco, arteria mesentérica superior, arterias renales, arteria mesentérica inferior con la columna vertebral.

En el caso del tronco celíaco se describieron sus ramas clásicas, arteria hepática común, arteria esplénica y ar- 
teria gástrica izquierda, determinando además, la ausencia de alguna de ellas y las respectivas variaciones.

Para el caso del nacimiento de la arteria mesentérica inferior se dividió el cuerpo de la vértebra correspondiente en tercios, debido al mayor tamaño de éstos respecto de los cuerpos vertebrales de otros sectores y a que su localización exacta es de implicancia clínica.

Las imágenes se analizaron computacionalmente para hacer un registro en planillas, donde para cada paciente se registraron las variables a estudiar. Se obtuvieron fotografías de cada uno de los registros tomográficos.

\section{RESULTADOS}

Tronco celíaco. El tronco celíaco se observó en 45 casos $(60,8 \%)$ con la clásica división en tres ramas, a. gástrica izquierda, a. hepática y a. esplénica; en $28(37,8 \%)$ estuvo formado por la a. hepática y a. esplénica. No se evidenció la arteria hepática en un caso $(1,3 \%)$.
La relaciones entre el origen de esta arteria y los cuerpos vertebrales, tanto en hombres como en mujeres, son mostradas en la Tabla I.

Arteria mesentérica superior. En todos los casos analizados se encontró una sola a. mesentérica superior. Su origen relacionado a los cuerpos vertebrales, tanto en hombres como en mujeres, se muestra en la Tabla II.

Arterias renales. En todos los casos estudiados (100\%) se encontró a. renal en ambos lados. Se observó doble en 14 casos (18,9\%, Figs. 1 y 2). Esta variación se presentó en 8 mujeres y 6 hombres. La relación con la columna vertebral, tanto en hombres como en mujeres, se muestra en la Tabla III. No se encontraron personas monorrenas en el estudio. En 12 casos las 2 arterias renales se originaron en relación al mismo cuerpo vertebral y en 2 casos en relación con vértebras sucesivas.

Arteria mesentérica inferior. Esta arteria se observó en todos los pacientes (100\%), localizando su origen en relación con la columna vertebral con la disposición que se muestra en la Tabla IV. En la Figura 3 se observa la relación del origen de la arteria mesentérica inferior con el tercio superior de la vértebra L4.

Tabla I. Niveles de origen del tronco celíaco respecto a los cuerpos vertebrales en hombres y mujeres.

\begin{tabular}{lcccc}
\hline Vértebra & Hombres & Mujeres & \multicolumn{2}{c}{ Total } \\
\hline & & & $\mathrm{N}^{\mathbf{2}}$ & $\%$ \\
T12 & 20 & 18 & 38 & 51 \\
L1 & 20 & 16 & 36 & 49 \\
\hline Total & 40 & 34 & 74 & 100 \\
\hline
\end{tabular}

Tabla II. Origen de la arteria mesentérica superior en relación con los cuerpos vertebrales en hombres y mujeres.

\begin{tabular}{ccccc}
\hline Vértebra & Hombres & Mujeres & \multicolumn{2}{c}{ Total } \\
\hline & & & $\mathrm{N}^{\mathbf{2}}$ & $\%$ \\
T12 & 4 & 2 & 6 & 8,1 \\
L1 & 32 & 26 & 58 & 78,4 \\
L2 & 4 & 6 & 10 & 13,5 \\
Total & 40 & 34 & 74 & 100 \\
\hline
\end{tabular}

Tabla III. Emergencia de las arterias renales en relación con la columna vertebral.

\begin{tabular}{lcccccc}
\hline Vértebra & Hombres & \multicolumn{2}{c}{ Mujeres } & \multicolumn{2}{c}{ Total } \\
\hline L1 & Lado derecho & Lado izquierdo & Lado derecho & Lado izquierdo & $\mathrm{N}^{\mathrm{o}}$ & $\%$ \\
L2 & 23 & 22 & 19 & 15 & 79 & 48,8 \\
L3 & 18 & 22 & 19 & 19 & 78 & 48,1 \\
L4 & 0 & 0 & 2 & 2 & 4 & 2,5 \\
\hline Total & 0 & 1 & 0 & 0 & 1 & 0,6 \\
\hline
\end{tabular}




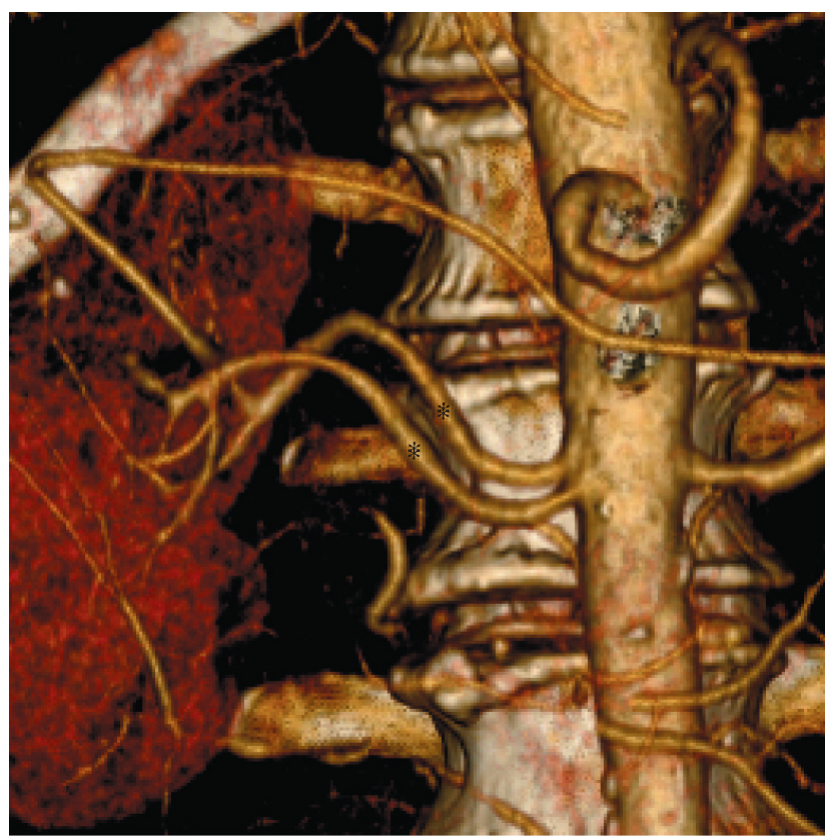

Fig. 1. Doble arterial renal (*) en el lado derecho.

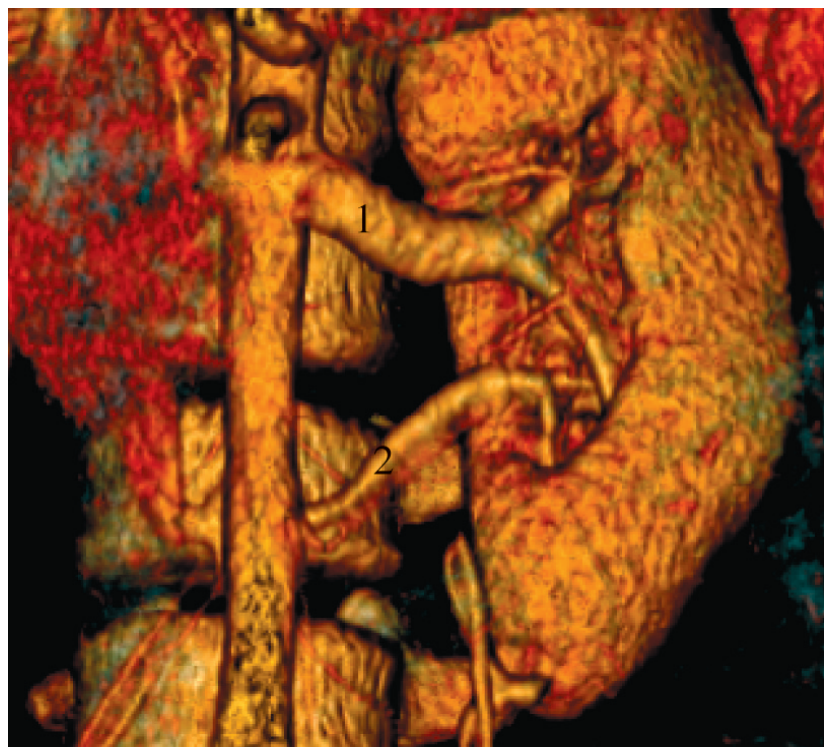

Fig. 2. Doble arteria renal (1 y 2 ) en el lado izquierdo, con orígenes a distinto nivel.

\section{DISCUSIÓN}

El conocimiento del sistema arterial y sus variaciones es de suma importancia para la cirugía en general, considerando que en la actualidad, con el advenimiento de terapias cada vez menos invasivas y con procedimientos diagnósticos, quirúrgicos y terapéuticos que requieren de mayor precisión y accesos mínimos, es necesario tener un aca-

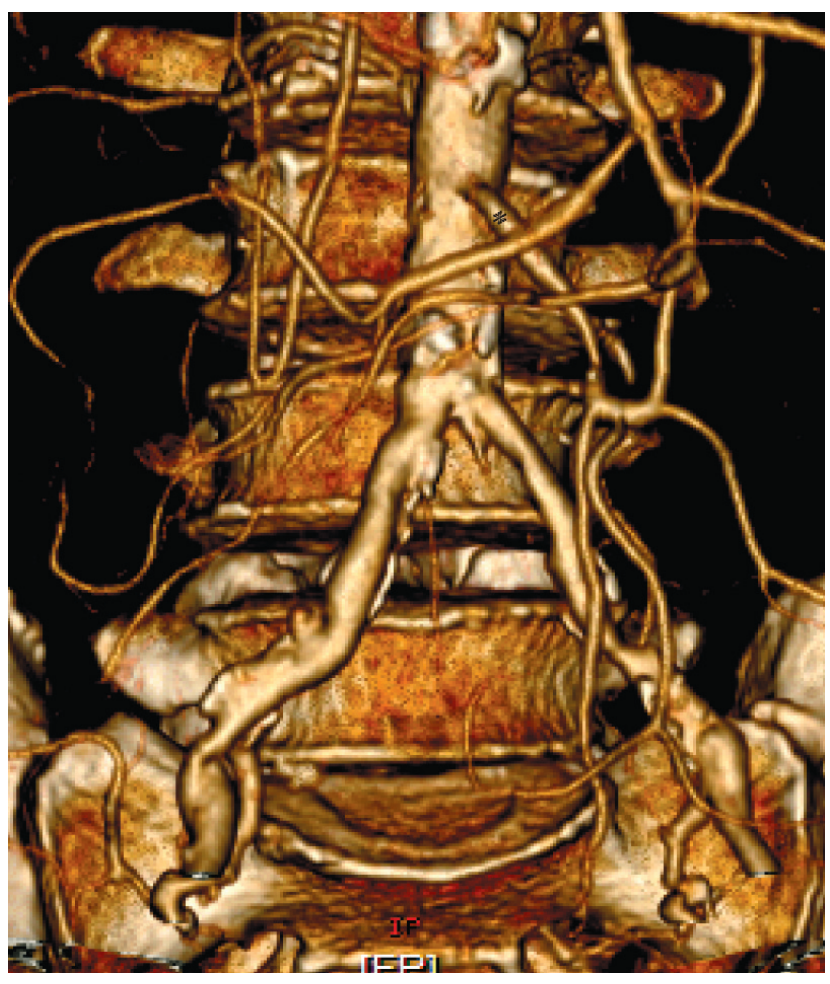

Fig. 3. Origen de la arteria mesentérica inferior (*) a nivel del tercio superior de la vértebra L3.

bado conocimiento anatómico de la posición, relaciones y ramas de los diferentes vasos del organismo.

Las nuevas tecnologías para obtener imágenes del cuerpo de individuos vivos, nos permiten acceder con bastante precisión a las diversas estructuras que le componen, entre otras, al sistema vascular, por lo que en este trabajo se ha logrado relacionar con mucha exactitud, el origen de arterias viscerales de la porción abdominal de la aorta con la columna vertebral, entregando así datos observados en personas de nuestra población, considerando que la gran mayoría de los antecedentes que se tienen de estos vasos, corresponde a resultados obtenidos en grupos de otros países. La literatura clásica refiere que el nacimiento del tronco celiaco se encuentra relacionado con la vértebra T12 (McKears \& Owen, 1979; Testut \& Latarjet; Goss, 1976), pero en nuestro grupo se encontró a este nivel en el 51\% de los casos y el resto a nivel de L1, de tal manera que se puede indicar que este tronco arterial puede originarse, a nivel de una u otra vértebra. Comparando esta disposición entre sexos no se encontraron diferencias significativas.

Iezzi et al. (2008) encontraron en un $72,1 \%$ de su estudio, la conformación clásica del tronco celíaco, un tronco gastroesplénico en un 2,3\% y un tronco hepatoesplénico en un $2,7 \%$. En los datos obtenidos en nuestro grupo, se 
Tabla IV. Emergencia de la arteria mesentérica inferior en relación a los tercios de los cuerpos vertebrales lumbares en hombres y mujeres.

\begin{tabular}{|c|c|c|c|c|}
\hline \multirow{2}{*}{$\begin{array}{l}\text { Vértebra } \\
\text { Niveles columna }\end{array}$} & \multirow{2}{*}{ Hombres } & \multirow[t]{2}{*}{ Mujeres } & \multicolumn{2}{|c|}{ Total } \\
\hline & & & $\mathrm{N}^{\circ}$ casos & $\%$ \\
\hline Inferior L2 & 0 & 6 & 6 & 8,1 \\
\hline Superior L3 & 17 & 5 & 22 & 29,8 \\
\hline Medio L3 & 7 & 7 & 14 & 18,9 \\
\hline Inferior L3 & 10 & 8 & 18 & 24,2 \\
\hline Superior L4 & 6 & 6 & 12 & 16,2 \\
\hline Medio L4 & 0 & 0 & 0 & 0 \\
\hline Inferior L4 & 0 & 2 & 2 & 2,7 \\
\hline Total & 40 & 34 & 74 & 100 \\
\hline
\end{tabular}

puede observar que la conformación clásica se encontró en el $60,8 \%$ y solo un tronco gastro-esplénico en un $1,3 \%$ de los casos. Estas variaciones de frecuencia pueden ser explicadas por diferencias raciales o por variaciones habituales del árbol arterial. La trifurcación clásica ha sido encontrada en diversos porcentajes (Katsume et al., 1978, 94,2\%; Nelson et al., 1988, 82\%). La división en dos ramas fue observada por estos mismos autores en $5,8 \%$ y $18 \%$, respectivamente. En nuestra serie encontramos la división de este tronco en arteria hepática y esplénica en un $37,8 \%$. Por su parte Yi et al. (2008a) encontraron dos troncos comunes independientes originándose de la aorta, uno gastrofrénico (arterias frénica inferior izquierda ygástrica izquierda) y el otro hepatoesplénico (arterias hepática común y esplénica). Recientemente, Raikos et al. (2010) publicaron un caso de tronco común freno-gastro-esplénico, donde la arteria hepática común se originó directamente de la aorta. Su ausencia también ha sido reportada (Vandamme \& Bonte, 1985; Yi et al, 2008b).

Ferrari et al. (2007) observaron en un 56,7\% de los casos estudiados, la conformación clásica del tronco celíaco, hallazgos con los que concordamos.

Pudimos constatar que en todos los pacientes se encontró la presencia de la a. mesentérica superior naciendo de la cara anterior de la aorta en relación con el cuerpo vertebral de L1 en un $78,4 \%$, superior a estudio semejante de Olave et al. (2009) que observaron esta disposición en 51,7\% y a nivel del disco intervertebralentre T12 y L1 en 9,7\%, tal vez reflejado por menor número de pacientes (31). En la literatura encontramos referencia de origen de ésta arteria en el tronco celíaco (Shaeffer, 1942; Testut \& Latarjet; Goss) disposición no encontrada en este estudio.

Con respecto a las arterias renales, han tenido un in- terés especial, en su estudio anatómico, ya que son asiento importante de patologías como las estenosis y en los últimos años en el estudio que de ellas se hace para el transplante renal de donantes vivos. El conocimiento del número y distribución de ellas resulta fundamental para realizar tales procedimientos. La literatura muestra diversos artículos donde se presentan arterias renales múltiples (dobles, triples e incluso de mayor número) en porcentajes variables 11,2\% (Wozniak, 2000); 18\% (Khamanarong et al., 2004); $20 \%$ (Dhar \& Lai, 2005). En nuestro estudio observamos arteria renal doble en el 18,9\% de los casos, coincidente con los autores mencionados. Son varios los reportes de casos con más de una arteria renal (Loukas et al., 2005; Tanyeli et al., 2006; Deepthinath et al., 2006; Olave et al., 2007). Por su parte, Khamanarong et al. mencionaron un $1 \%$ de arterias triples, lo que no se encontró en nuestro estudio.

Respecto a los niveles de origen de las arterias renales, relacionando a éste con la columna vertebral, son varios los estudios en serie realizados en diversos países, como también se han mostrado variaciones del mismo, entre las cuales se pueden mencionar: un origen común con la arteria mesentérica superior (Dalçik et al., 2000), origen alto de la arteria renal derecha (Fernet et al.,1987), origen asociado con otras ramas de la aorta abdominal (Bakheit \& Motabagani, 2003).

Los niveles encontrados en nuestro grupo de estudio, corresponden en porcentajes muy similares, a los cuerpos vertebrales lumbares L1 $(48,8 \%)$ y L2 $(48,1 \%)$; no observamos nacimientos más altos como los descritos por Fernet et al., quienes lo visualizaron a nivel del disco entre T11 y T12, coincidentes con lo señalado por Ozkan et al. (2006) quienes describieron porcentajes similares en cuanto a la relación con L1 y L2, señalando de forma general que el origen de las arterias renales se encuentra entre el 
margen superior de la vértebra L1 y el margen inferior de la vértebra L2 en $98 \%$ de sus casos ( 855 pacientes), disposición semejante a nuestros resultados (lado derecho $97,5 \%$ y lado izquierdo 96,3\%). Estos últimos porcentajes son un poco mayores que los datos obtenidos por Olave et al. en 2009 (90,3\% y $87,1 \%$, respectivamente). Por su parte, Lee et al. (2004) señalaron que en 83 pacientes (39\%) de 210 estudiados, el origen de esta arteria fue a nivel de la vértebra L1, diferente de nuestros resultados donde el nacimiento de este vaso se efectuó a nivel de L1 en 48,8\%.

Respecto a la arteria mesentérica inferior, ésta se encuentra presente y única en el $100 \%$ de la población chilena y la relación de su origen con el cuerpo vertebral, corresponde en la mayoría de los casos a L3, en sus diversos sectores, lo que corresponde a descripciones clásicas (Testut \& Latarjet), origen que McKears \& Owen lo describen entre las vértebras L2 y L3. Sin embargo, la observamos también con orígenes más altos, a nivel de L2 y más bajos, en relación a L4, disposición no encontrada en la literatura consultada. Las variaciones anatómicas de esta arteria son raras, pero se han descrito casos donde este vaso se ha originado desde la arteria mesentérica superior (Osawa et al., 2004; Yi et al., 2008a) o de un tronco común con la arteria mesentérica superior a nivel de la vértebra L5 (Maleux et al., 2010).

La variabilidad encontrada en cuanto al número, conformación y las relaciones de los elementos vasculares estudiados es un hecho a tener en cuenta para los diferentes actos médicos que involucren intervención directa del operador.

La disponibilidad de nuevas tecnologías de imagen cada vez más precisas en cuanto a la capacidad de discriminar entre densidades de tejidos diferentes, se conocen y se han desarrollado sólo desde fines del siglo pasado y se han enfocado preferencialmente al estudio de patologías arteriales y su información como método diagnóstico y previo a terapias definitivas (Zeman et al., 1994).

La posibilidad de hacer relaciones entre los diferentes órganos y tejidos mediante estas técnicas es lo que mejor se destaca en los resultados obtenidos. Estas imágenes permiten su estudio acabado y reproducible y no son influenciables por fenómenos cadavéricos, derivados de una disección defectuosa y son prácticamente inocuos para la persona a estudiar.

Este tipo de imágenes obtenida con tomografía helicoidal multidetectores tiene, entre otras ventajas, una gran velocidad para la toma de imágenes, fina sincronización en la utilización del medio de contraste y un potente software informático para procesar las imágenes. Todo ello hace posible un aumento de la resolución temporo-espacial y un deta- lle anatómico que solo se logra en preparaciones de alta calidad

No existen estudios publicados en nuestro medio con esta metodología, que describan las características anatómicas normales de la aorta abdominal y sus ramas, que permitan comparar los resultados obtenidos, ya que en su mayoría se describen patologías vasculares, lo que no es el objetivo del presente estudio.

La utilización de la tomografía con multidetectores cambia dramáticamente la posibilidad de estudiar la anatomía de las estructuras vasculares con los órganos y tejidos que lo rodean (Wintersperger et al., 2004). Esta tecnología es la que se utilizó en este trabajo, lo que nos permitió hacer descripciones detalladas y reproducibles de las características y relaciones estudiadas.

Sin duda que la posibilidad de contar con estas imágenes vasculares y sus relaciones tridimensionales facilitan los procedimientos quirúrgicos invasivos o métodos diagnósticos que requieren localizar los elementos vasculares.

El conocimiento exacto de la anatomía vascular por el método estudiado, sin duda permite al cirujano plantear una estrategia adecuada de enfrentamiento a la patología del paciente, sobre todo en aquellas personas sanas como los donantes vivos de órganos.

La visualización tridimensional rotatoria que permite esta técnica de obtención de imágenes, permite describir las relaciones entre órganos o estructuras, que solo se obtienen en preparados y disecciones muy laboriosas, y esta tecnología logra esto en solo minutos y con riesgo mínimo para el paciente.

Sin duda que esta investigación deja abierta la puerta para obtener rica información sobre la anatomía vascular, diámetros, relaciones entre si, variaciones y otras que serán abordadas en próximas investigaciones.

PUELMA, F. \& OLAVE, E. Relationships between of the origin of celiac trunk, mesenteric and renal arteries with the vertebral column in Chilean individuals. Int. J. Morphol., 28(4):1227$12 x x, 2010$.

SUMMARY: At present many surgical invasive, diagnostic and therapeutic procedures require a precise knowledge of the arterial anatomy of the abdominal territory. Among these interventions the extraction of organs stands out in alive donors (renal and hepatic) and the oncological interventions, among others. Traditionally, the study of the vascularization has been carried out by means of arteriographies which require invasive procedures with 
injection of contrast substances and long expossue to X-rays, obtaining two-dimensional images in which the relations between the tissues and organs is difficult to analyze. The aim of the present work was to determine the anatomical characteristics of the celiac trunk, superior mesenteric, renal and inferior mesenteric arteries and the relation of origin with the vertebral column. Images of 74 patients, 40 men and 34 women, submitted to an tomography of the abdominal part of the aorta, were studied as were the characteristics and the relations of the mentioned arteries. Images used were obtained by means of multidetector computed tomography. The classic division of the celiac trunk in three branches was observed in $60,8 \%$ of the cases, the origin related with $\mathrm{T} 12$ in $50 \%$ of the men and in 52,9\% of the women, with L1 in $50 \%$ and $47,1 \%$, respectively. The superior mesenteric artery originated to level of T12, L1 and L2 in 8,1 \%, 78,4\% and 13,5\%, respectively. The renal arteries were double in $18,9 \%$ of the cases. With regard to the classic descriptions we found differences that demonstrate the variability of the vascular distribution. The method is validated as a useful instance of low risk for the patient and reproducible for the researchers and clinical.

KEY WORDS: Anatomy; Abdominal part of the aorta, Multidetector computed tomography.

\section{REFERENCIAS BIBLIOGRÁFICAS}

Bakheit, M.A. \& Motabagani, M.A. Anomalies of the renal, phrenic and suprarenal arteries: case report. East Afr. Med. J., 80(9):497-8, 2003.

Beebe, H.G. \& Kritpracha, B. Imaging of abdominal aortic aneurysm: current status. Ann. Vasc. Surg., 17:11-8, 2003.

Cikrit, D.F.; Harris, V.J. ; Hemmer C.G. ; Kopecky, K. ; Dalsing, M.; Hyre, C.; Fischer, JM.; Lalka, SG. ; Sawchuk. Comparison of spiral CT scan an arteriography for evaluation of renal and visceral arteries. Ann. Vasc. Surg., 10:109-16, 1996.

Dalcik, C.; Colak, T.; Ozbek, A.; Dalcik, H. Unusual origin of the right renal artery: a case report. Surg. Radiol. Anat., 22(2):117-8, 2000.

Deepthinath, R.; Satheesha Nayak, B.; Bhat, S.; Rodriguez, V.; Samuel, V.; Venkatamarana, V. \& Prasad, A.M. Multiple variations in the paired arteries of the abdominal arteries. Clin. Anat., 19(6):566-9, 2006.

Dhar, P. \& Lai, K. Main and accessory renal arteries-a morphological study. Ital. J. Anat. Embryol., 110(2):10110, 2005.

Fernet, M.; Goldlust, D.; Salama, J.; Chevrel, J.P. A case of ectopic renal artery: a radiologic-anatomic variant. Surg. Radiol. Anat., 9(4):319-20, 1987.
Ferrari, R.; De Cecco, C.N.; Iafrate, F.;Paolantonia, P.; Rengo, M. \& Laghi, A. Anatomical variations of the celiac trunk and mesenteric arteries evaluated with 64-row CT angiography. Radiol. Med., 112(7):988-98, 2007.

Goss, Ch. Gray Anatomía. 29a ed. Salvat, Barcelona, 1976.

Iezzi R.; Cotroneo, A.R.; Giancristofaro, D.; Santoro, M. \& Sorto, M. L. Multidetector-row CT angiographic Imaging of the celiac trunk: anatomy and normal variation. Surg. Radiol. Anat., 30 (4): 303-10, 2008.

Katsume, K.; Kanamura, E.; Sakai, K.; Yoshizuka, M.; Hirotzu, A. \& Ishibashi, K. apud Yi, S-Q.; Li, J.; Terayama, H.; Naito, M.; Hirai, S.; Alimujang, S.; Yi, N.; Tanaka, S. \& Itoh, M. Absence of the celiac trunk: Case report and review of the literature. Clin. Anat., 21:283-6, 2008.

Khamaranong, K.; Prachaney, P.; Utravarichien, A.; Tongun, T. \& Sripoaraya, K.; Anatomy of renal artery supply. Clin. Anat., 17 (4):334-6, 2004.

Lee, C. H.; Seo, B. K.; Choi, Y. Ch.; Shin, H. J.; Park, J. H.; Jeon, H. J.; Kim, K. A.; Park, Ch. M. \& Kim, B. H. Using MRI to evaluate anatomic significance of aortic bifurcation, right renal artery, and conus medullaris when locating lumbar vertebral segments. AJR, 182:1295-300, 2004.

Loukas, M.; Aparicio, S.; Beck, A.; Calderon, R. \& Kennedy, M. Rare case of accessory renal artery originating as a common trunk with the inferior mesenteric artery. A case report. Clin. Anat., 18(7): 530-5, 2005.

Maleux, G.; Vaninsbroukx, J.; Demedts, I.; Heye, S. Common trunk of superior and inferior mesenteric artery at the level of the fifth lumbar vertebra. J. Vasc. Interv. Radiol., 21(2):296-8, 2010.

Mautone, A. \& Brown, J.R. Contrast-Induced nephropathy in patient undergoing elective and urgent procedures. $J$. Interv. Cardiol., 23(1):78-85, 2010.

McKears, D.W. \& Owen, R.H. Surface Anatomy for Radiographers. Bristol, John Wright \& Sons Ltd., 1979.

Moore KL.; Dalley AF. Anatomía con Orientación Clínica. $4^{\mathrm{a}}$ Ed. Buenos Aires: Panamericana, 2004.

Nelson, T. M.; Pollak, R.; Jonasson, O. \& Abcarian, H. Anatomics variants of the celiac, superior mesenteric and inferior mesenteric arteries and their clinical relevance. Clin. Anat., 1:75-91, 1988. 
Olave, E.; Henriquez, G.; Cruzat, C.; Puelma, F.\& Soto, A. Arterias renales múltiples. Int. J. Morphol., 25(4): 92730, 2007.

Olave, E.; Puelma, F.; Henriquez, G.; Cruzat, C. \& Soto, A. Niveles de origen de las arterias renales y mesentérica superior respecto de la columna vertebral en individuos chilenos. Estudio por tomografía computarizada helicoidal. Int. J. Morphol.,27(2):447-52, 2009.

Osawa, T.; Feng, X.Y.; Sasaki, N.; Nagato, S.; Matsumoto, Y.; Onodera, M.; Nara, E.; Fujimura, A. \& Nozaka, Y. Rare case of the inferior mesenterio artery and the common hepatic artery arising from the superior mesneteric artery. Clin. Anat., 17(6):518-21, 2004.

Ozkan, U.; Oguzkurt, L.; Tercan, F.; Kizilkilic, O.; Koc, Z. \& Koca, N. Renal artery origins and variations: angiographic evaluation of 885 consecutive patient. Diagn. Interv. Radiol., 12(4):183-6, 2006.

Raikos, A.; Paraskevas, G.K.; Natsis, K.; Tzikas, A. \& Njau, S.N. Multiple variations in the branching pattern of the abdominal aorta. Rom. J. Morphol. Embryol. 51(3):5857, 2010 .

Rubin GD.; Dake MD.; Napel S.; McDonnell, C.H. \& Jeffrey R.B. Three-dimensional spiral CT angiography of the abdomen initial clinical experience. Radiology, 186:147-52, 1993a.

Rubin, G. D.; Walker P. J.; Dake, MD.; Napel, S.; Jeffrey, R. B.; McDonnell, C. H.; Mitchell, R. S. \& Miller. Threedimensional spiral computed tomographic: an alternative imaging modality for de abdominal aorta and its branches. J.Vasc. Surg., 665: 656-64, 1993 b.

Schaeffer, J.P. Morris'Human Anatomy. $11^{\mathrm{a}}$ ed. New York, McGraw Hill, 1942.

Tanyeli, E.; Uzel, M. \& Soyluoglu, A.L. Complex renal vascular variation, a case report. Ann. Anat., 188(5): 4558, 2006.

Testut, L. \& Latarjet, A. Tratado de Anatomía Humana. Barcelona, Salvat, 1969.

Tooher, R.L.; Rao, M.M.; Scott, D.F.; Wall, D.R.; Francis, D.M.; Bridgewater, F..H. \& Maddern, G.J. A systematic review of laparoscopic live-donor nephrectomy. Transplantation, 78(3):404-14, 2004.

Tovar, S.; Ernesto, R.; Peña, M.A.; Luna, J.; Vásquez, J. \& Martínez, M.A. Translumbar coronary and aortography in patient with Takayasu's arteritis. Arch. Inst. Cardiol. Mex., 69(2):149-52, 1999.

Vandamme, J.P. \& Bonte, J. The branches of the celiac trunk. Acta Anat (Basel), 122:110-4, 1985.

Wang, J.D.; Liu, Y.D.; Quan, Z.W. Li, S.G.; Wang, X.F. \& Sheh J. Role of regional lymphadenectomy in different stage of gallbladder carcinoma. Hepatogastroenterology, 56(91-92):593-6, 2009.

Williams, P. L.; Warwick, R.; Dyson, M.; Bannister, L. H. Gray Anatomía. 37 $7^{\text {th }}$ Ed. Rio de Janeiro, GuanabaraKoogan, 1995.

Wintersperger, B.J.; Nikolau, K. \& Becker, C.R. Multidetectorrow $\mathrm{CT}$ angiography of the aorta and visceral arteries. Semin. Ultrasound CT MR., 1:25-40, 2004.

Wozniak, W.T. Origin of the renal arteries from sides of aorta. Folia Morphol. (Warsz), 58(4):259-61, 2000.

Yi, S-Q.; Li, J.; Terayama, H.; Naito, M.; Iimura, A. \& Itoh, M. A rare case of inferior mesenteric artery arising from the superior mesenteric artery, with a review of the review of the literature. Surg. Radiol. Anat., 30:159-65, 2008a.

Yi, S-Q.; Li, J.; Terayama, H.; Naito, M.; Hirai, S.; Alimujang, S.; Yi, N.; Tanaka, S. \& Itoh, M. Absence of the celiac trunk: Case report and review of the literature. Clin. Anat., 21:283-6, 2008b.

Zeman, R. K.; Silverman, P. M.; Berman, P. M.; Weltman, D. I.; Davros, W. J. \& Gomez, M. N. Abdominal aortic aneurysms: evaluation with variable collimation helical CT and over lapping reconstruction. Radiology, 193:55560, 1994.

Dirección para correspondencia:

Dr. Felipe Puelma

Facultad de Medicina

Universidad Católica del Maule

Talca CHILE

Email: fpuelma@ucm.cl

Recibido: 11-08-2010

Aceptado:14-09-2010 